\title{
Breast Carcinoma; Human Epidermal Growth Factor Receptor-2 (HER-2) and Grading Correlation
} \author{
Tabatabaee $^{4}$, Yekta Parsa ${ }^{5,}$, , Soheila Yadollah-Damavandi ${ }^{5}$, Tina Parsa ${ }^{5}$, Amir Aidun ${ }^{6,7}$ \\ ${ }^{1}$ Department of Surgery, Islamic Azad University, Tehran Medical Sciences Branch, Tehran, Iran \\ ${ }^{2}$ Department of General, Visceral, and Transplantation Surgery, University of Heidelberg, Heidelberg, Germany \\ ${ }^{3}$ Department of Surgery, Shahid Beheshti University of Medical Sciences, Tehran, Iran \\ ${ }^{4}$ Students' Research Committee, Islamic Azad University, Tehran Medical Sciences Branch, Tehran, Iran \\ ${ }^{5}$ Young Researchers and Elite Club, Islamic Azad University, Tehran Medical Sciences Branch, Tehran, Iran \\ ${ }^{6}$ Department of Biomedical Engineering, Amirkabir University of Technology, Tehran, Iran \\ ${ }^{7}$ National Cell Bank of Iran, Pasteur Institute of Iran, Tehran, Iran
}

Seyed Abbas Mirmalek ${ }^{1}$, Fatemeh Elham Kani', Arianeb Mehrabi ${ }^{2}$, Mehdi Chavoshi-Nejad ${ }^{3}$, Seyed Alireza Salimi-

*Corresponding Author: Yekta Parsa, Young Researchers and Elite Club, Islamic Azad University, Tehran Medical Sciences Branch, Attarimoqaddam Ave, Haqani Ave, Dr. Shariati St, Tehran, Iran. Email: yektaparsa@gmj.ir

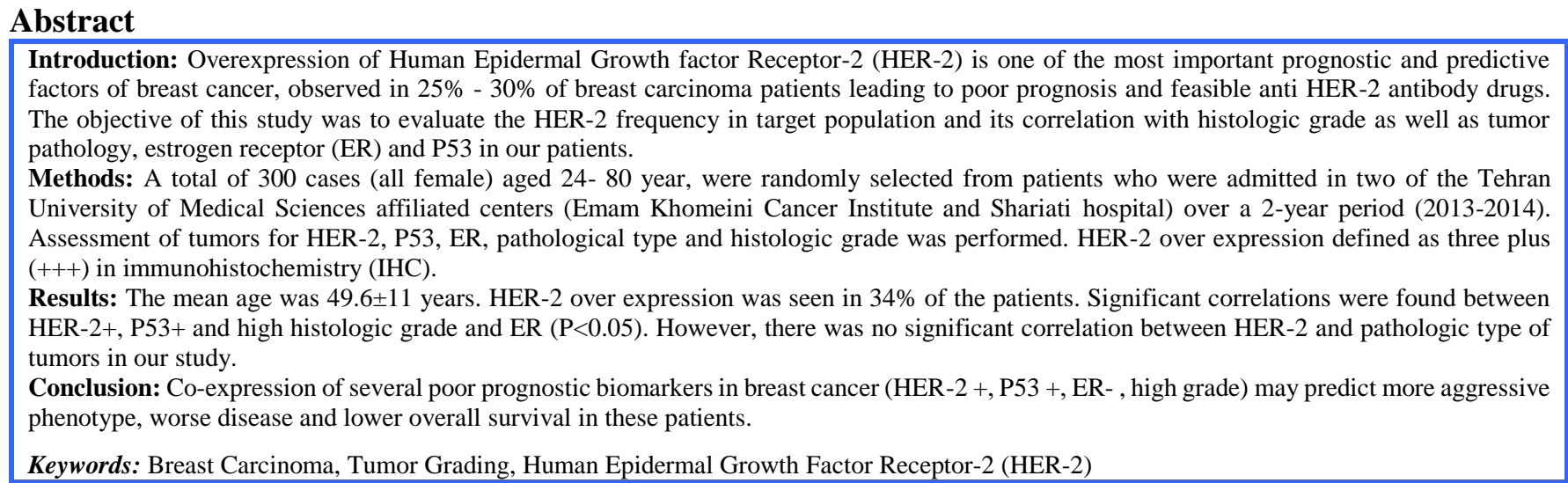

Article History: Received: 20 Feb. 2015; Accepted: 6 Apr. 2015; Online Published: 24 Aug. 2015

Cite this article as: Mirmalek SA, Elham Kani F, Mehrabi A, Chavoshi-Nejad M, Salimi-Tabatabaee SA, Parsa Y, et al. Breast carcinoma; human epidermal growth factor receptor-2 (her-2) and grading correlation. Int J Travel Med Glob Health. 2015;3(3):95-7.

\section{Introduction}

Breast cancer is the most common invasive cancer in women, including nearly $23 \%$ of invasive cancers and $16 \%$ of all female cancers. The incidence, $18-78$ per 100,000 women, varies greatly around the world, lowest in lessdeveloped and greatest in the more-developed countries. Primary risk-factors include female sex and older age. Other potential risk factors include genetics, lack of childbearing or lack of breastfeeding, higher levels of certain hormones, certain dietary patterns, and obesity. Furthermore, recent studies have indicated that exposure to air pollution is a risk factor for the development of breast cancer [1-3].

Along all other cancers, breast cancer is also categorized by the standard Grading and Staging method. Grading compares the appearance of the breast cancer cells to the appearance of normal breast tissues. Normal cells in an organ like the breast become differentiated, meaning that they take on specific shapes and forms that reflect their function as part of that organ. In cancer, the cells that would normally line up in an orderly way to make up the milk ducts become disorganized. Cell division becomes uncontrolled. Cell nuclei become less uniform. Pathologists describe cells as well differentiated (low grade), moderately differentiated (intermediate grade), and poorly differentiated (high grade) as the cells progressively lose the features seen in normal breast cells. Poorly differentiated cancers (the ones whose tissue is least like normal breast tissue) have a worse prognosis [4-5].

Furthermore, in the last 2 decades, several biomarkers have been founded to be associated with breast cancer. Chemical messengers such as hormones bind to receptors, and this causes changes in the cell. Breast cancer cells may or may not have three important receptors: Estrogen Receptor (ER), Progesterone Receptor (PR), and Human Epidermal growth factor Receptor-2 (HER-2) [1-5].

$\mathrm{ER}+$ cancer cells depend on estrogen for their growth, so they can be treated with drugs to block estrogen effects (e.g. Tamoxifen), and generally have a better prognosis. Untreated, HER-2+ breast cancers are generally more aggressive than HER-2- breast cancers, but HER-2+ cancer cells respond to drugs such as the monoclonal antibody Trastuzumab (in combination with conventional chemotherapy), and this has improved the prognosis significantly. Cells that do not have any of these three receptor types (estrogen receptors, progesterone receptors, or HER-2) are called triple-negative, although they frequently do express receptors for other hormones, such as androgen receptor and prolactin receptor [6-8].

Therefore, regarding the great importance of breast cancer prognosis and its known relation with HER-2, and furthermore, regarding the newly introduced chemotherapy targeting HER-2, the aim of this study was to evaluate HER- 
2 frequency in target population and its correlation with histologic grade as well as tumor pathology, ER, and P53 in our patients.

\section{Methods}

\subsection{Subjects}

This is an analytical cross sectional study; conducted on all patients, complaining of breast lumps, and following by a pathology-confirmed diagnosis of breast carcinoma, to the surgery day clinic of Tehran University of Medical Sciences affiliated hospitals (Imam Khomeini and Shariati hospital, Tehran, Iran) from December 2013 to December 2014. Using the registration data, 1502 patients were enrolled; however, 686 individuals were included in our random selection by excluding patients with incomplete information. Furthermore, patients with other chronic diseases, other types of diagnosed carcinomas and other breast pathologies were also excluded from our study. Regarding the sample size calculation formula for finding a proportion, [n=z2 $\mathrm{P}(1-$ $\mathrm{P}) / \mathrm{d} 2$ ] and standard constants (alpha $=0.05$ and beta=0.2) a proportion of $0.5 \%$ for breast cancer and $25 \%$ prevalence of HER-2 positive according to WHO reports, 300 patients were randomly selected. Demographic information was collected through computer database. Regarding ethical issues and research standards, all patients who participated in this research provided written consent forms.

\subsection{Tumor Characteristics}

The HER-2 was evaluated by immunohistochemistry (IHC) method according to the fluorescent staining. All sections were categorized into four subgroups: negative, + , ++ and +++ ). In our study, patient with HER-2 level of +++ was considered as positive. Furthermore, ER and P53 situations were also evaluated by different IHC methods, categorizing them into positive and negative states.

We used histologic differentiation to categorize the severity of the disease. Two expert pathologists evaluated all the sections and the histologic grade of the tumor was recorded according to Bloom and Richardson differentiation [9]: well differentiated, moderate differentiated and poorly differentiated. Furthermore, all pathology reports included the type of carcinoma: invasive ductal carcinoma (IDC) and invasive lobular carcinoma (ILC). Other rare types of cancers including, in-situ forms, tubular and papillary carcinomas were excluded from our data collection.

\subsection{Statistical Analysis}

Data analysis was done by Statistical Package for the Social Sciences (SPSS, Chicago, Illinois, USA) software version 11.5. Normality of data was checked by ShapiroWilk test. Student t-test and Mann-Whitney test were done for the comparison of quantitative variables. Qualitative variables between the two groups were compared by Chisquare test. $\mathrm{P}$ value less than 0.05 was considered to be statistically significant.

\section{Results}

The population of this study consisted of 300 women with a mean age of $49.6 \pm 10.86$ years (minimum 24 and maximum 80 years-old) referred to Imam Khomeini and Shariati Hospitals. Totally, 102 patients (34\%) were HER-2 positive, $193(65.3 \%)$ were ER positive and $121(40.3 \%)$ were P53 positive. The pathology report consisted of 298 (99.3\%) of IDC and only 2 patients suffering from ILC. In addition, 34 out of 300 included cancer pathologies were at Grade I, 144 $(48 \%)$ in grade II and $126(42 \%)$ in grade III.
Results demonstrated that significant differences was found between HER-2 positivity and tumor grade (Grade I, II and III: $13.3 \%, 38.2 \%$ and $34.1 \%$, respectively; $p=0.033$ ). Significant differences was also found between grade I with grades II and III. However, grade II and grade III HER-2 positivity rate did not differ significantly. Significant difference was found in ER and P53 positivity and HER-2 positivity: $48.6 \%$ ER positive vs. $25.9 \%$ ER negative in HER-2 positive patients, $\mathrm{p}<0.001 ; 43 \%$ P53 positive vs. $27.9 \%$ P53 negative in HER-2 positive patients, $\mathrm{p}=0.007$.

For better analysis, we categorized patients in two groups: grade I, and grade "II and III". In Logistic regression analysis in patients with positive HER-2, the rate of grade II and III had a 6.2 fold higher prevalence (CI: 1.831 - 21.138, $\mathrm{p}=0.003$ ) in P53 positive patients. However, in HER-2 negative patients, the Odd's Ratio was 3 (CI: $1.126-9.197$, $\mathrm{p}=0.045$ ). Our results did not demonstrate any significant predictive correlations between ER and HER-2.

\section{Discussion}

Our study was a prevalence description of known pathology's biomarkers of breast cancer in 2 cancer referral centers in Iran. Regarding our results, HER-2, P53 and ER positivity increase, relating to cancer grades. The main results were in accordance to other similar studies worldwide. However, as we did not have a baseline description in the Iranian breast cancer society - as far as our knowledge - the aim of this study was to evaluate HER-2 frequency in the target population and its correlation with histologic grade, tumor pathology, ER and P53.

According to the high prevalence of breast cancer in women and its negative impact on their physical and psychological life, improving prognosis has a high importance in these patients. In addition, estimating life expectancy can play an important role in answering patients. Therefore, finding a way to estimate patients' prognosis is one of the most important fields of research; pathologic and serum biomarkers as the first to explore $[10,11]$. E-erb B2, i.e. HER-2, was discovered to be related to breast cancer in 1989, demonstrating that higher HER-2 expression correlate with poorer prognosis and higher rates of recurrence [7, 8]. Some chemotherapy drugs such as Tanstuzumab target HER-2 are effecting in controlling breast cancer. However, these drugs can only act on tumors with high presentation of HER-2 on malignant cells. Tests are usually performed on biopsy samples obtained by fine-needle aspiration, core needle biopsy, vacuum-assisted breast biopsy, or surgical excision.

Immunohistochemistry is used to measure the amount of HER-2 protein present in the sample. Alternatively, fluorescence in situ hybridisation (FISH) can be used to measure the number of copies of the gene which are present [8].

The prognostic impact of HER-2 positivity is lower in node-negative compared with node-positive women. HER2-positive tumors also contain p53 abnormalities, tending to be hormone receptor and bcl-2 negative, have lymphoid infiltration (LI) and a high mitotic index. Patients with LI who are HER-2 positive have a better prognosis than those who are HER-2 negative, whereas HER-2 positive patients without LI have a significantly worse prognosis than HER-2 negative patients. Morphological and biological alterations appear to identify categories of breast tumors [5-7].

There are several studies conducted regarding $\mathrm{ICH}$ 
biomarkers in breast cancer. In a study conducted in Alabama University, USA, a 15-year follow-up demonstrated that over expression of HER-2 and P53 is highly associated with breast cancer's clinical and pathological outcome [12]. Another study conducted in Japan in 2003 demonstrated co-expression of HER-2 and showed that P53 can better predict patients' outcome comparing with only one positive biomarker [13]. Both studies demonstrated that higher HER-2, ER and P53 expression is correlated with higher tumor grades. Furthermore, an Australian [14] and a Spanish [15] study demonstrated higher Biomarker expression is associated with higher tumor grade; which represents that higher HER2, ER and P53 tumors accounts for poorer prognosis.

The most important study regarding HER-2 expression in Iranian Breast cancer was conducted by Keyhani et al [16]. In this review article 22 studies were included and 3,033 patients were evaluated, 1,350 were diagnosed as HER-2positive by IHC. The mean percentage of HER-2-positive patients was $44.5 \%$, which is higher than that recorded in international statistics. IHC HER-2 testing has been performed in Iran for over 10 years. Similar to many other countries, before establishment of an infrastructure for IHC diagnostic tests, HER-2 testing was routinely performed in Iran. Our study showed that the statistics reported from Iran varied widely; for instance, the rate of HER-2positive cases varied from $23.3 \%$ to $81.0 \%$.

\section{Conclusion}

The objective of this study was to evaluate HER-2 frequency in target population and its correlation with histologic grade as well as tumor pathology, ER and P53 in our patients. The study shows that $34 \%$ of Iranian cancer patients were HER-2 positive. Significant relation was found between HER-2 positivity with ER and P53. In Logistic regression analysis in patients with positive HER-2, the rate of Grade II and III had a 6.2 fold higher prevalence in P53 positive patients; however, in HER-2 negative patients, the Odd's Ratio was 3.

\section{Acknowledgments}

Authors would like to express their sincere thanks to Dr. Ehsan Jangholi for his cooperation and insightful comments on this manuscript.

\section{Authors' Contributions}

Study concept and design: SAM, FEK and AM. Acquisition of data: AA, YP and TP. Analysis and interpretation of data: SARST, SYD and MCJ. Drafting of the manuscript: YP, SYD and MCJ. Critical revision of the manuscript for important intellectual content: TP and AA.
Statistical analysis: SARST and AM.

\section{Financial Disclosure}

The authors declare that there is no financial disclosure regarding the publication of this paper.

\section{Funding/Support}

This study was supported by the Islamic Azad University, Tehran Medical Sciences Branch.

\section{References}

1. Ban KA, Godellas CV. Epidemiology of Breast Cancer. Surg Oncol Clin N Am. 2014;23(3):409-422.

2. Mirmalek SA, Elhamkani F, Tabatabaee SAS, Mahmoodzadeh H, Parsa Y, Yadollah-Damavandi S, et al. Introduction of HER-2 and a Short Review on Its Role in Prognosis and Treatment of Breast Cancer. GMJ. 2014;3(3):132-44.

3. Brewster A, Helzlsouer K. Breast cancer epidemiology, prevention, and early detection. Curr Opin Oncol. 2001;13(6):420-5

4. Carlson RW, Allred DC, Anderson BO, Burstein HJ, Carter WB, Edge SB, et al. Breast cancer. Clinical practice guidelines in oncology. J Natl Compr Canc Netw. 2009;7(2):122-92.

5. Sotiriou C, Pusztai L. Gene-expression signatures in breast cancer. N Engl J Med. 2009;360(8):790-800.

6. Romond EH, Perez EA, Bryant J, Suman VJ, Geyer CE Jr, Davidson NE, et al. Trastuzumab plus adjuvant chemotherapy for operable HER2-positive breast cancer. N Engl J Med. 2005;353(16):1673-84.

7. Mitri Z, Constantine T, O'Regan R. The HER2 Receptor in Breast Cancer: Pathophysiology, Clinical Use, and New Advances in Therapy. Chemother Res Pract. 2012; 2012:743193.

8. Burstein HJ. The distinctive nature of HER2-positive breast cancers. N Engl J Med. 2005;353(16):1652-4.

9. Howell LP, Gandour-Edwards R, O'Sullivan D. Application of the Scarff-Bloom-Richardson tumor grading system to fine-needle aspirates of the breast. Am J Clin Pathol. 1994;101(3):262-5.

10. Najmabadi KM, Azarkish F, Latifnejadroudsari R, Shandiz FH, Aledavood SA, Kermani AT, Esmaily HO. Self-disclosure of breast cancer diagnosis by Iranian women to friends and colleagues. Asian Pac J Cancer Prev. 2014;15(6):2879-82

11. Bell K. The breast-cancer-ization of cancer survivorship implications for experiences of the disease. Soc Sci Med 2014;110:56-63.

12. Nowsheen S, Cooper T, Bonner JA, LoBuglio AF, Yang ES. HER2 overexpression renders human breast cancers sensitive to PARP inhibition independently of any defect in homologous recombination DNA repair. Cancer Res. 2012;72(18):4796-806.

13. Chen HH, Su W-C, Guo H-R, Chang T-W, Lee W-Y. p53 and cerbB-2 but not bcl-2 are predictive of metastasis-free survival in breast cancer patients receiving post-mastectomy adjuvant radiotherapy in Taiwan. Jpn J Clin Oncol. 2002;32(9):332-9.

14. Bilous M, Ades C, Armes J, Bishop J, Brown R, Cooke B, et al. Predicting the HER2 status of breast cancer from basic histopathology data: an analysis of 1500 breast cancers as part of the HER2000 International Study. The breast. 2003;12(2):92-8.

15. López-Guerrero JA, Llombart-Cussac A, Noguera R, Navarro S, Pellin A, Almenar S, et al. HER2 amplification in recurrent breast cancer following breast-conserving therapy correlates with distant metastasis and poor survival. Int J Cancer. 2006;118(7):1743-9.

16. Keyhani E, Muhammadnejad A, Karimlou M.Prevalence of HER-2 positive invasive breast cancer: a systematic review from Iran. Asian Pac J Cancer Prev. 2012;13(11):5477-82. 